\title{
Sources of fatty acids stored in liver and secreted via lipoproteins in patients with nonalcoholic fatty liver disease
}

\author{
Kerry L. Donnelly, ${ }^{1}$ Coleman I. Smith, ${ }^{2,3}$ Sarah J. Schwarzenberg, ${ }^{4}$ \\ Jose Jessurun, ${ }^{5}$ Mark D. Boldt, ${ }^{3}$ and Elizabeth J. Parks ${ }^{1,6}$
}

\begin{abstract}
1Department of Food Science and Nutrition, University of Minnesota, Twin Cities, St. Paul, Minnesota, USA. 2Department of Medicine, Division of Gastroenterology, Hepatology and Nutrition, University of Minnesota, Twin Cities, Minneapolis, Minnesota, USA. ${ }^{3 M i n n e s o t a ~ G a s t r o e n t e r o l o g y, ~ P . A ., ~ S t . ~ P a u l, ~}$ Minnesota, USA. ${ }^{4}$ Department of Pediatrics, Division of Gastroenterology, Hepatology and Nutrition, ${ }^{5}$ Department of Laboratory Medicine and Pathology, and ${ }^{6}$ Department of Medicine, Division of Endocrinology and Diabetes, University of Minnesota, Twin Cities, Minneapolis, Minnesota, USA.
\end{abstract}

\begin{abstract}
Nonalcoholic fatty liver disease (NAFLD) is characterized by the accumulation of excess liver triacylglycerol (TAG), inflammation, and liver damage. The goal of the present study was to directly quantify the biological sources of hepatic and plasma lipoprotein TAG in NAFLD. Patients (5 male and 4 female; $44 \pm 10$ years of age) scheduled for a medically indicated liver biopsy were infused with and orally fed stable isotopes for $\mathbf{4}$ days to label and track serum nonesterified fatty acids (NEFAs), dietary fatty acids, and those derived from the de novo lipogenesis (DNL) pathway, present in liver tissue and lipoprotein TAG. Hepatic and lipoprotein TAG fatty acids were analyzed by gas chromatography/mass spectrometry. NAFLD patients were obese, with fasting hypertriglyceridemia and hyperinsulinemia. Of the TAG accounted for in liver, $59.0 \% \pm 9.9 \%$ of TAG arose from NEFAs; $26.1 \% \pm 6.7 \%$, from DNL; and $14.9 \% \pm 7.0 \%$, from the diet. The pattern of labeling in VLDL was similar to that in liver, and throughout the 4 days of labeling, the liver demonstrated reciprocal use of adipose and dietary fatty acids. DNL was elevated in the fasting state and demonstrated no diurnal variation. These quantitative metabolic data document that both elevated peripheral fatty acids and DNL contribute to the accumulation of hepatic and lipoprotein fat in NAFLD.
\end{abstract}

\section{Introduction}

Nonalcoholic fatty liver disease (NAFLD) is a term used to describe a spectrum of conditions characterized mainly by the histological finding of macrovesicular hepatic steatosis in individuals who consume little or no alcohol (1). The classic NAFLD patient has persistently elevated liver enzymes (aspartate aminotransferase [AST] and alanine aminotransferase [ALT]); has negative test results for viral, congenital, and autoimmune liver diseases; and denies excessive alcohol consumption (2). Previously, fatty liver was thought to be benign. However, it has recently become clear that fatty liver is a precursor of the more advanced liver disease nonalcoholic steatohepatitis, a condition that may progress to cirrhosis in up to $25 \%$ of patients $(3,4)$. Fatty liver is now considered to be the hepatic component of the metabolic syndrome (5-8). The specific origin of the lipids that accumulate in liver remains unknown, and identifying the origin of the accumulated lipids in the livers of patients with NAFLD will be important in the prevention and reversal of this condition.

The hallmark feature of the pathogenesis of NAFLD, both histologically and metabolically, is the accumulation of triacylglycerol (TAG) in the liver. The plasma nonesterified fatty acid (NEFA)

\footnotetext{
Nonstandard abbreviations used: ALT, alanine aminotransferase; AST, aspartate aminotransferase; DNL, de novo lipogenesis; GC/MS, gas chromatography/mass spectrometry; GCRC, General Clinical Research Center; $\mathrm{HOMA}_{\mathrm{IR}}$, homeostasis model assessment of insulin resistance; MIDA, mass isotopomer distribution analysis; NAFLD, nonalcoholic fatty liver disease; NEFA, nonesterified fatty acid; RaNEFA, rate of appearance of NEFA; TAG, $\mathrm{S}_{\mathrm{f}}$, Svedberg flotation unit; triacylglycerol; tTRL, total TAG-rich lipoprotein.

Conflict of interest: The authors have declared that no conflict of interest exists.

Citation for this article: J. Clin. Invest. 115:1343-1351 (2005).

doi:10.1172/JCI200523621.
}

pool contributes the majority of the fatty acids that flow to the liver in the fasted state and thus provides the bulk of the fatty acids secreted by the liver in VLDL particles (9). Insulin resistance has been shown to be associated with dysregulation of adiposederived fatty acid flux in the fasting state (10-12), and strong evidence exists demonstrating that in NAFLD patients, insulin does not suppress adipose tissue lipolysis to the same extent that it does in healthy individuals (13).

Yet other sources of fatty acids can contribute to fatty liver, and an understanding of fatty acid regulation in the fasted and fed states should take these sources into account. The potential sources of fats contributing to fatty liver are shown in Figure 1 and include peripheral fats stored in adipose tissue that flow to the liver by way of the plasma NEFA pool (pathway 1); fatty acids newly made within the liver through de novo lipogenesis (DNL) (pathway 2); and dietary fatty acids, which can enter the liver by two means: through spillover into the plasma NEFA pool (14) (pathway 3) and through the uptake of intestinally derived chylomicron remnants (15) (pathway 4). Isotope methodology has been used to quantify fatty acid $(9)$ and TAG $(9,16,17)$ flux in the fasting state in healthy, obese, and hypertriglyceridemic patients, and in one study of NAFLD (18). Using the multiple-stable-isotope approach, we have recently developed a new method to determine the fate of dietary fatty acids during the postprandial state $(19,20)$, and in the present work we have combined these techniques with liver biopsy to simultaneously measure all four pathways of fatty acid delivery to the liver in patients suspected of having NAFLD. These quantitative metabolic data demonstrated that both elevated peripheral fatty acid flux and DNL contribute to hepatic and lipoprotein fat in NAFLD. 


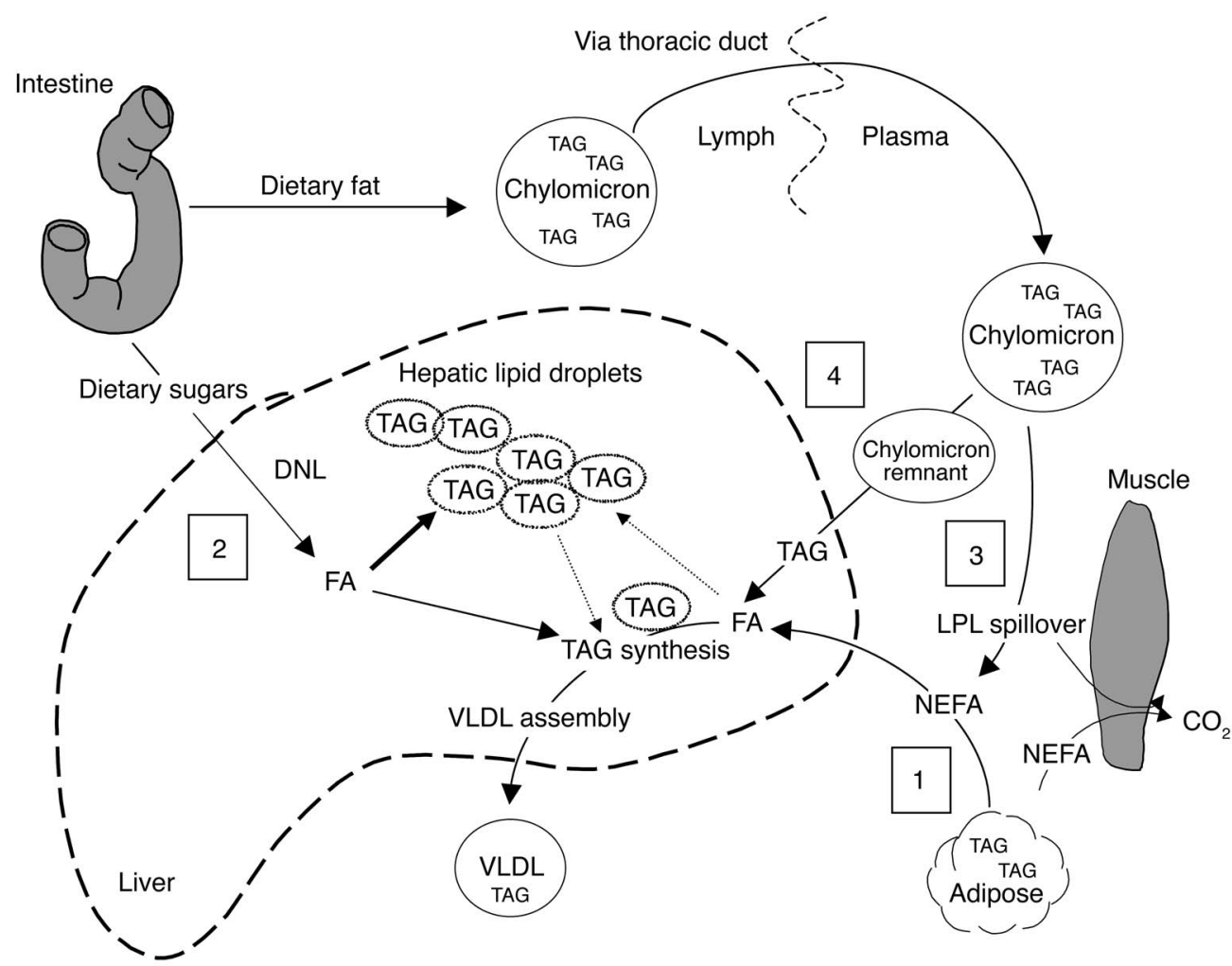

\section{Figure 1}

Model of lipid flux through the liver. Boxed numbers indicate the metabolic pathways traced using stable (nonradioactive) isotopes. DNL indicates new fat synthesis from 2-carbon precursors (e.g., dietary carbohydrate); chylomicrons are lipoproteins made in the intestine that carry dietary fat. FA, cellular fatty acids (fatty acids not esterified to glycerol but bound to a carrier protein); LPL, lipoprotein lipase.

\section{Results}

Clinical and laboratory values of the subjects ( 5 male and 4 female; $44 \pm 10$ years of age) revealed characteristics common to NAFLD patients (Table 1). As determined by computed tomography scans, both visceral and subcutaneous fat masses were elevated above the normal values reported by Dixon (21). Serum ALT was elevated in all subjects, serum AST was elevated in most subjects, and in most subjects, serum alkaline phosphatase was within the normal range (data not shown). In addition, fasting serum TAG concentrations revealed hypertriglyceridemia $(3.2 \pm 2.2 \mathrm{mmol} / \mathrm{l})$ and the homeostasis model assessment of insulin resistance $\left(\mathrm{HOMA}_{\mathrm{IR}}\right)$, a quantitative index of insulin resistance (22), was also elevated (4.1 \pm 2.8 ; range, $1.2-8.9)$. The first goal of the present study was to assess the hepatic response during transitions from fasting to feeding with respect to how fatty acids from difference sources were used for lipoprotein TAG synthesis. In the fasting state on days 2,3 , and 4, the isolation of large buoyant VLDL (Svedberg flotation unit $\left.\left[\mathrm{S}_{\mathrm{f}}\right], 60-400\right)$ revealed an average fasting concentration of $1.0 \pm 0.9 \mathrm{mmol} / \mathrm{l}$. The rate of appearance of NEFA (RaNEFA) on day 2 was $6.3 \pm 2.9 \mu \mathrm{mol} / \mathrm{kg} / \mathrm{min}$. The remaining data presented at the bottom of Table 1 provide the metabolic context in which lipoprotein TAG metabolism was determined and demonstrate the expected differences between fasting and feeding (e.g., higher insulin concentrations in the fed state, lower NEFA, etc.). Changes in fatty acid sources in blood lipids are shown in Figure 2. In both states, the majority of serum NEFA originated in adipose tissue (Figure 2A). After 4 days of labeling, the average contributions of adipose fatty acids to the serum NEFA pool were $81.7 \% \pm 8.2 \%$ and $61.7 \% \pm 11.9 \%$ for fasted and fed states, respectively (mean \pm SD; $P=0.0003$ ). The spillover of dietary TAG into the NEFA pool was evident on the very first afternoon after subjects were fed the labeled TAG at lunch and contributed $16.3 \% \pm 8.2 \%$ of these fatty acids. The fed-state contribution of dietary lipid to serum NEFA rose to $26.2 \% \pm 8.9 \%$ after 4 days of labeling, a value that was significantly higher than the fasting contribution on day $5(10.4 \% \pm 4.9 \%$; $P=0.0006)$. For newly made fatty acids, those in the serum NEFA pool could have originated from DNL in adipose or liver. The contribution of DNL to serum NEFA was lower in the fasting state than in the fed state $(7.0 \% \pm 4.0 \%$ versus $9.4 \% \pm 4.5 \% ; P=0.01)$. The sources of fatty acids in total TAG-rich lipoproteins (tTRLs) were analyzed for comparison, and the pattern of labeling in lipoprotein TAG mirrored that of the serum NEFA pool (Figure 2B). After 4 days of labeling, the contribution of adipose- and dietderived fatty acids to tTRL TAG synthesis changed significantly from fasting to feeding, with adipose-derived fatty acids being higher in the fasting state $(60.4 \% \pm 18.2 \%$ and $27.9 \% \pm 10.2 \%$, respectively; $P=0.0001)$ and diet-derived fatty acids being higher in the fed state $(12.1 \% \pm 4.1 \%$ versus $19.1 \% \pm 8.3 \% ; P=0.01)$. In contrast, the contribution of DNL to tTRL TAG did not differ between fasting and feeding $(22.2 \% \pm 8.1 \%$ versus $20.4 \% \pm 5.2 \%$; $P=0.29)$. At every time point, the serum NEFA pool contributed the most to tTRL TAG, regardless of metabolic state $(P=0.001)$. Evidence of recycling of the isotopes was suggested by the slow rise in sources apparent over the 4 days. The contributions of NEFA and newly made fatty acids to tTRL TAG increased with time $(P=0.007)$, while the contribution of dietary fatty acids to tTRL TAG showed a trend toward increasing with time $(P=0.06)$.

Changes in the sources of tTRL TAG immediately after consumption of a meal. Given previous observations of higher fed-state DNL (23) and the known stimulatory effect of insulin on fatty acid synthesis (24), we were surprised that fed-state lipogenesis assessed at 1500 in tTRL TAG did not differ from values obtained in the fasting 
Table 1

Clinical and laboratory values in NAFLD patients

\begin{tabular}{|c|c|c|c|}
\hline $\begin{array}{l}\text { Subject characteristics } \\
(n=9)\end{array}$ & Mean \pm SD & Range & \\
\hline Body weight $(\mathrm{kg})$ & $108.2 \pm 17.0$ & $85.8-136.4$ & \\
\hline BMI $\left(\mathrm{kg} / \mathrm{m}^{2}\right)$ & $36 \pm 5$ & $29-44$ & \\
\hline Total body fat (\%) & $44.7 \pm 11.1$ & $30.2-55.9$ & \\
\hline Visceral fat $\left(\mathrm{cm}^{2}\right)$ & $231 \pm 50$ & $192-324$ & \\
\hline Subcutaneous fat $\left(\mathrm{cm}^{2}\right)$ & $496 \pm 178$ & $213-723$ & \\
\hline AST (U/I) & $68 \pm 34$ & $63-186$ & \\
\hline \multirow[t]{2}{*}{$\operatorname{ALT}(\mathrm{U} / \mathrm{l})$} & $102 \pm 41$ & $29-147$ & \\
\hline & Fasted & Fed & $P$ value \\
\hline Serum insulin $(\mathrm{pmol} / \mathrm{l})^{\mathrm{A}}$ & $24.8 \pm 12.9$ & $117.6 \pm 131.4$ & 0.006 \\
\hline Serum glucose $(\mathrm{mmol} / \mathrm{l})^{\mathrm{A}}$ & $5.6 \pm 0.8$ & $6.2 \pm 0.8$ & 0.036 \\
\hline $\mathrm{NEFA}(\mathrm{mmol} / \mathrm{l})^{\mathrm{A}}$ & $0.55 \pm 0.33$ & $0.34 \pm 0.24$ & 0.001 \\
\hline tTRL TAG $(\mathrm{mmol} / \mathrm{l})^{\mathrm{A}}$ & $1.7 \pm 1.8$ & $2.2 \pm 2.3$ & 0.032 \\
\hline $\mathrm{EE}(\mathrm{kcal} / \mathrm{kg} / \mathrm{min})^{\mathrm{B}}$ & $0.012 \pm 0.002$ & $0.014 \pm 0.002$ & 0.001 \\
\hline$N P R Q^{B}$ & $0.83 \pm 0.07$ & $0.84 \pm 0.07$ & 0.509 \\
\hline $\begin{array}{l}\text { Fat oxidation } \\
\qquad(\mathrm{mg} / \mathrm{kg} / \mathrm{min})^{B}\end{array}$ & $0.58 \pm 0.29$ & $0.55 \pm 0.32$ & 0.589 \\
\hline $\begin{array}{l}\text { Glucose oxidation } \\
(\mathrm{mg} / \mathrm{kg} / \mathrm{min})^{\mathrm{B}}\end{array}$ & $0.94 \pm 0.59$ & $1.02 \pm 0.52$ & 0.495 \\
\hline
\end{tabular}

Data in the upper panel are baseline data obtained during screening procedure. NAFLD patients were on various medications, including medications for hypertension, hypothyroidism, and depression. One subject was being treated for diabetes. ${ }^{\mathrm{A}}$ Fasted and fed values represent the average concentrations obtained on days 2, 3, and 4 at 0645 (fasted) and 1500 (fed) for each subject. BValues were calculated from data collected on day 4 of the inpatient protocol from 0600 to 0630 for fasting and from 1500 to 1530 for fed data. EE, energy expenditure; NPRQ, non-protein respiratory quotient.

state. To determine if fed-state data collected at 1500 each day were truly reflective of metabolism after a meal, subjects underwent a meal test (breakfast meal). As shown in Figure 2C, these data demonstrate that the contribution of NEFA to tTRL TAG decreased from a peak of $55.6 \% \pm 4.3 \%$ to a nadir of $32.4 \% \pm 2.9 \%(P=0.0004)$. The contribution of dietary TAG to tTRL TAG increased slightly after the meal (peaking 3 hours after the meal; $P=0.02$ ) to a value that did not differ from the percentage found 3 hours after lunch $(P=0.77)$. After consumption of breakfast, DNL did not change from fasting nor were postprandial values different from the value 3 hours after lunch $(P=0.77)$.

As described in Methods, VLDL particles were isolated from all blood drawn from subjects in the fasting state. For comparison with the tTRL, this is represented by unfilled symbols on Figure $2 \mathrm{~B}$. In the fasting state, the sources of VLDL TAG were similar to those in tTRLs. The rate of accrual of DNL fatty acids was faster than the rate of accrual for dietary fatty acids $(3.5 \%$ per day versus $1.7 \%$ per day; $P=0.004)$. This faster rate of accumulation resulted in a greater contribution of de novo fatty acids than of dietary fatty acids to VLDL TAG $(22.4 \% \pm 8.2 \%$ and $13.2 \% \pm 6.5 \%$, respectively; $P=0.01$ ). The final labeling patterns of lipoprotein TAG are summarized in Figure $3 \mathrm{~A}$ and demonstrate the constant contribution of lipogenesis in the fasted and fed states. The contribution of total dietary fatty acids to VLDL TAG was calculated to be greater than could be accounted for by entry through the serum NEFA pool. These fatty acids, assumed to have entered the liver via chylomicron remnant uptake, represented $7.2 \% \pm 5.6 \%$ of VLDL TAG in the fasting state. The inability to account for all of the sources of fatty acids used for tTRL TAG synthesis in the fed state (Figure 3A) may have been due to a greater use of unlabeled fatty acids for VLDL TAG synthesis, since dietary sources (and hence chylomicrons in this fraction) would have been labeled. This observation is consistent with a greater appearance of unlabeled fatty acids apparent in VLDL TAG found in the early postprandial period in our studies of healthy subjects (M.T. Timlin and E.J. Parks, unpublished observations).

On the fifth day of the study, each subject underwent a liver biopsy, and the histological results of this procedure are presented in Table 2 (also included for comparison are subject ages and $\mathrm{BMI}$ ). The TAG content of liver tissues ranged from not detectable by gas chromatography (below $1 \mu \mathrm{mol}$ fatty acid) to $0.49 \mathrm{~g}$ per gram of tissue, which was higher than the $0.01 \mathrm{~g}$ per gram of tissue typically observed in healthy populations $(25,26)$. Histological evaluation of liver biopsies indicated steatosis in all subjects, inflammation in all but 2 subjects, and progression to fibrosis in 4 subjects (Table 2). In the liver, labeling with stable isotopes over 5 days allowed for the identification of $20.4-63.7 \%$ of hepatic TAGpalmitate. No physical or biochemical characteristics correlated with the percentage of liver TAG labeled over this period. There was, however, a strong correlation between the percentage of TAG fatty acid sources accounted for in liver and those accounted for in VLDL (Figure 3B), and a weak association between the TAG content of liver and the percentage of liver TAG accounted for by the labeling protocol $\left(r^{2}=0.46 ; P=0.15\right)$. The fractional synthesis rate of the total liver TAG pool ranged from $5.1 \%$ to $15.5 \%$ per day and thus the average time estimated for the near-complete turnover of this pool was $38.4 \pm 15.6$ days (Table 2 ). Although not significant, the turnover time tended to be associated with the liver TAG content $\left(r^{2}=0.37 ; P=0.11\right)$. To compare sources of liver TAG and TAG secreted in VLDL, we normalized the sources contributing to both TAG pools to $100 \%$ (Figure $3 \mathrm{~A}$ ). In VLDL and liver TAG, no differences between the proportions of the three fatty acid sources were found. Fatty acids originating in the NEFA pool contributed $62.4 \% \pm 11.7 \%$ and $59.0 \% \pm 9.9 \%$, respectively, with newly made fatty acids contributing $22.9 \% \pm 6.2 \%$ and $26.1 \% \pm 6.7 \%$, respectively, and dietary fats contributing $14.7 \% \pm 8.5 \%$ and $14.9 \% \pm 7.0 \%$, respectively. Although NEFA represented the major source of liver TAG, the normalized values ranged from $45.1 \%$ to $74.3 \%$. The variability in the use of newly made fatty acids was $12.7 \%$ to $37.0 \%$, with the dietary contribution ranging from $4.3 \%$ to $28.0 \%$.

\section{Discussion}

The accumulation of fat, mainly TAG, within the hepatocyte is a prerequisite for the development of NAFLD. However, the primary metabolic abnormalities leading to lipid accretion are not well understood. Various strategies to treat NAFLD are currently focusing on improving glucose tolerance and body weight status (27-31); however, an understanding of the origins of the accumulated TAG in the liver will strengthen current treatment regimens and aid in the identification of new targets for therapy. Using a multiple-stable-isotope approach in patients who underwent a liver biopsy for diagnosis of their condition, we have documented here the sources of hepatic and lipoprotein TAG fatty acids, which included serum NEFA, newly made fatty acids, and dietary fatty acids. The key contributions of this work are that it (a) clearly demonstrated the dominance of serum NEFA in liver TAG pools in NAFLD; (b) clarified the effect of insulin resistance manifested through increased rates of DNL that fail to respond to nutritional state; (c) expanded 

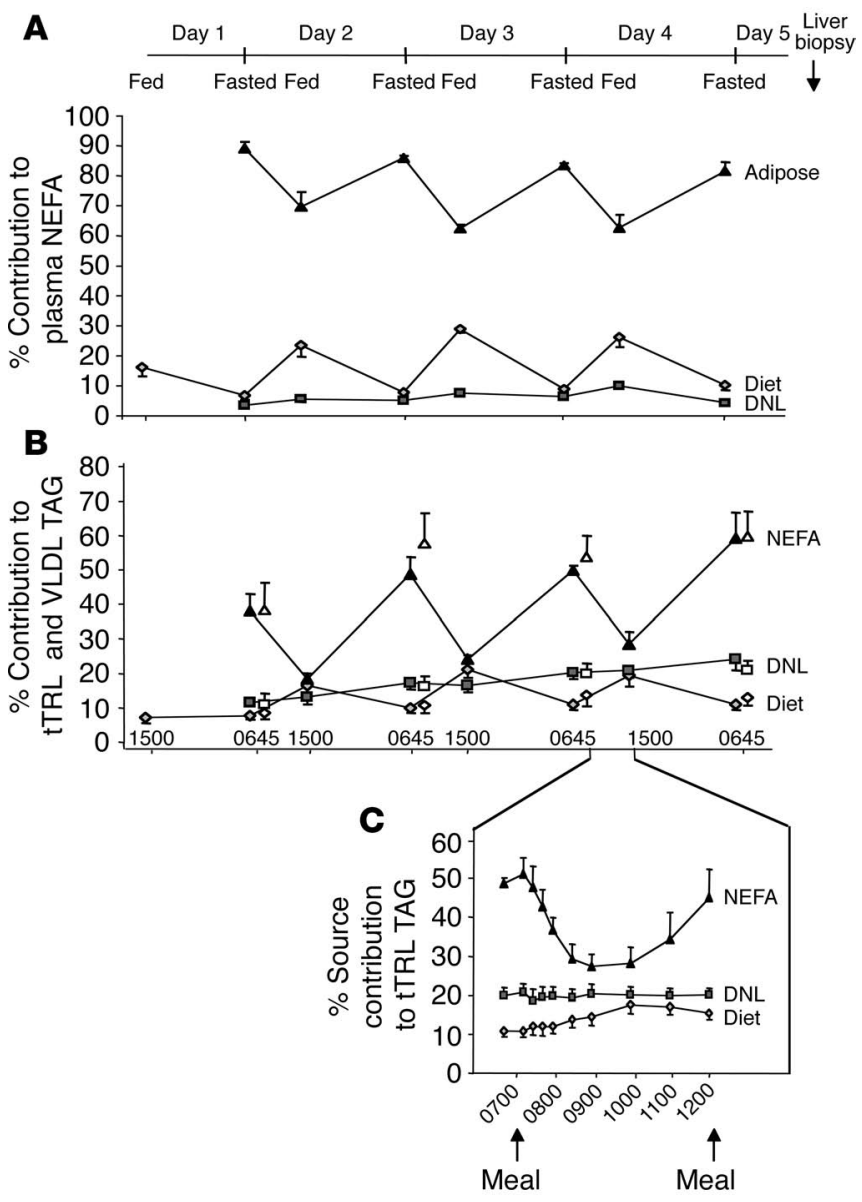

the view of the liver's diurnal control of fatty acid metabolism, with particular emphasis on the contribution of dietary fatty acids to human liver TAG pools; and (d) demonstrated for the first time to our knowledge the similarity between the fatty acid sources that contribute to hepatic TAG and lipoprotein TAG.

The first key finding of our research here was that the serum NEFA pool was the primary contributor to hepatic TAG in the fasting state and to lipoprotein TAG in both the fasting and fed states. Sanyal et al. found elevated insulin concentrations failed to suppress adipose fatty acid flux in NAFLD (13), a result that suggested that this source of fatty acids would be important to the development of fatty liver. Indeed, NAFLD patients treated with insulin-sensitizing agents show marked improvement in their condition $(28,30,31)$. Given that the majority of liver fatty acids were derived from the serum NEFA pool, therapeutic strategies should continue to focus on the reduction of adipose tissue fatty acid flux. The second key finding of our research was that DNL was elevated in the fasting state and failed to increase postprandially. In the fasting state, $26 \%$ of liver TAG and $23 \%$ of VLDL TAG fatty acids were derived from DNL, indicating that roughly one-fourth of all the fatty acids accounted for had been newly made from 2-carbon precursors derived from glucose, fructose, and amino acids. This elevation in fasting VLDL TAG from DNL in our subjects was higher than the $15 \%$ shown by Diraison and colleagues (18), a result most likely due to our longer duration of labeling and from the use of a lipoprotein fractionation procedure that isolated buoyant VLDL particles, representative of those most recently secreted by the liver.

\section{Figure 2}

Fatty acid sources in the fasted and fed states. (A and B) Sources of serum NEFA (A) and sources of tTRL TAG (filled symbols in B) were analyzed in the fasted and fed states. Sources of VLDL TAG (open symbols in B) were analyzed in the fasted state only. Data for fasting measurements were collected at 0645; fed-state concentrations were obtained 3 hours after lunch (1500) on each day. All data are mean \pm SE; $n=9$. (C) Insert shows the source contribution to tTRL TAG in the acute postprandial period. Patients consumed a meal consisting of whole foods at 0700; blood samples were obtained at 15-minute intervals until 0800, at 30-minute intervals until 0900, and then hourly until 1200 .

The presence of a large amount of lipoprotein remnants in hypertriglyceridemic individuals would tend to dilute apparent DNL in labeling studies less than 6 hours in duration. The 24\% DNL observed here is twice the $10 \%$ reported previously in obese, hyperinsulinemic subjects (32) and is 4-5 times the less than 5\% observed in healthy populations $(33,34)$. We have also recently documented a pattern of significant stimulation of lipogenesis in healthy subjects after consumption of a meal, rising from less than $5 \%$ to a peak of $23 \%$ within 4 hours of both the first morning meal and lunch (23). For NAFLD patients, the absence of this diurnal pattern of DNL was suggested in the daily measurements (Figure 2B) and was confirmed with a breakfast test (Figure 2C). Schwarz and colleagues found elevations in fasting DNL in obese, hyperinsulinemic subjects consuming ad libitum (high-fat) diets and demonstrated that those subjects failed to respond in the same manner as healthy subjects when switched to chronic consumption of a high-carbohydrate diet (32). The healthy subjects' lipogenesis rates tripled when measured in the fasting state after 5 days of high-carbohydrate feeding, while no change was observed in the fasting lipogenic rate of insulin-resistant subjects consuming the same diet (32). We have shown here that chronic consumption of a high-carbohydrate diet is not needed to detect this defect, as our NAFLD patients exhibited elevated fasting DNL and failed to appropriately increase lipogenesis postprandially when fed a meal consisting of $30 \%$ fat. The lack of postprandial stimulation suggests the liver's lipogenic potential may be reaching a threshold in these patients.

A third key finding of our studies relates to the significant extent to which the liver manages its use of the different sources of fatty acids that flow to it throughout the day. Inspection of Figure 2 demonstrates the reciprocal nature of these fluxes, such that during fasting, the hepatic use of dietary fatty acids became reduced and the deficit was made up by adipose tissue-derived fatty acids. It is well known that postprandially, dietary fatty acids spill over into the plasma NEFA pool (35), and we have shown here that their use for lipoprotein TAG synthesis rose and fell systematically. We have recently measured the extent to which dietary fatty acids escape peripheral storage in adipose tissue (by the combination of spillover and liver remnant uptake) in healthy subjects $(36,37)$, and our findings have confirmed that in NAFLD, this source can contribute substantially (approximately $15 \%)$ to both liver and VLDL TAG. It is likely that the dietary contribution to both TAG pools would be larger if dietary fat consumption had been higher than $30 \%$ of daily energy requirements. Data from mice in our lab confirm that this is the case in animals $(19,38)$. Collectively, these data suggest that the liver's system of fatty acid usage handles fluctuations that result in changes of up to $60 \%$ of the fatty acids used for VLDL TAG synthesis from fasting to feeding. 
Table 2

Liver function and histological evaluation of NAFLD patients

\begin{tabular}{|c|c|c|c|c|c|c|c|c|c|c|}
\hline \multirow[b]{2}{*}{ Subject } & \multicolumn{6}{|c|}{ Histological grading ${ }^{A}$} & \multirow[b]{2}{*}{$\begin{array}{c}\% \text { TAG } \\
\text { labeled }\end{array}$} & \multirow[b]{2}{*}{$\begin{array}{l}\text { FSR }^{B} \\
(\% / d)\end{array}$} & \multirow[b]{2}{*}{$\begin{array}{l}\text { ASR } \\
(g / d)\end{array}$} & \multirow[b]{2}{*}{$\begin{array}{l}\text { Turnover } \\
\text { (d) }\end{array}$} \\
\hline & Age/gender & $\begin{array}{c}\text { BMI } \\
\left(\mathrm{kg} / \mathrm{m}^{2}\right)\end{array}$ & Steatosis & Inflammation & Fibrosis & $\begin{array}{c}\text { Liver TAG } \\
\text { (g/g tissue) }\end{array}$ & & & & \\
\hline 1 & $36 / \mathrm{M}$ & 29 & 3 & 1 & 0 & 0.11 & 20.4 & 5.1 & 3.9 & 62.6 \\
\hline 2 & $49 / F$ & 38 & 3 & 1 & 0 & 0.49 & 21.6 & 5.4 & 16.1 & 59.2 \\
\hline 3 & $36 / M$ & 43 & 1 & 2 & 2 & ND & 49.8 & ND & ND & ND \\
\hline 4 & $57 / \mathrm{F}$ & 39 & 3 & 1 & 1 & 0.09 & 30.0 & 7.5 & 4.4 & 42.6 \\
\hline 5 & $43 / \mathrm{M}$ & 30 & 2 & 0 & 0 & 0.05 & 56.5 & 14.1 & 4.9 & 22.6 \\
\hline 6 & $61 / \mathrm{M}$ & 37 & 3 & 0 & 0 & 0.09 & 36.4 & 9.1 & 5.3 & 35.1 \\
\hline 7 & $31 / \mathrm{F}$ & 44 & 3 & 2 & 2 & 0.04 & 63.7 & 15.9 & 5.3 & 20.1 \\
\hline 8 & $45 / \mathrm{M}$ & 32 & 3 & 2 & 1 & 0.21 & 38.5 & 9.6 & 15.8 & 33.2 \\
\hline 9 & $36 / F$ & 32 & 3 & 1 & 0 & 0.16 & 40.4 & 10.1 & 9.7 & 31.6 \\
\hline Mean $( \pm$ SD) & & $36(5)$ & $2.7(0.7)$ & $1.1(0.8)$ & $0.7(0.9)$ & $0.16(0.15)$ & $38.4(15.6)$ & $9.6(3.8)$ & $8.2(5.1)$ & $38.4(15.6)$ \\
\hline
\end{tabular}

AGraded according to Dixon (45). Steatosis grades: 1, less than 5\% of lobular parenchyma involved; 2, 5-25\%; 3, 25-75\%. Inflammation grades: 0, no inflammation; 1 , sparse; 2 , mild. Fibrosis grades: 0 , normal connective tissue; 1 , focal pericellular fibrosis, zone 3 ; 2 , perivenular and pericellular fibrosis, zones 2 and 3. BLiver turnover times were estimated using an assumed liver weight of $2,000 \mathrm{~g}(16)$ and an estimation of $5 \times t_{1 / 2}$ for near-complete turnover of the hepatic TAG pool (50). ASR, absolute synthesis rate; FSR, fractional synthesis rate; ND, not detectable (i.e., the fat content of the liver of subject 3 was below the detection limit of the gas chromatograph, and therefore no turnover time could be estimated).

The final and most novel finding of our work was a significant similarity between the contributions of fatty acid sources found in the liver TAG pools and the sources used for TAG secretion by the liver via lipoproteins. In many studies, labeling with a glycerol or fatty acid isotope has been used to investigate lipoprotein TAG synthesis rates (as reviewed previously by Baker in ref. 39 and more recently by Patterson in ref. 40), but we have found only 1 paper in the literature that combined labeling of VLDL TAG with analysis of liver tissue biopsy samples (16). Farquhar and colleagues used a fatty acid label in liver turnover studies of 4 men with normal liver TAG pool sizes (e.g., estimates between 3 and $11 \mathrm{~g}$ TAG/liver, equivalent to $0.4-1.0 \%$ wet weight of tissue). As only a single fatty acid label was used, no comparison could be made between the various fatty acid sources contributing to liver and lipoprotein TAG. In our study here, the similarity between the 2 TAG pools provides support for future studies in which lipoprotein fatty acid may be used as an indicator for the liver TAG fatty acid pool in order to determine the success of treatments designed to reduce hepatic TAG stores.

The fact that the rates of appearance of labeled fatty acids rose over the 5 days of study deserves some comment. We expected $(16,39)$ and observed recycling of fatty acids over this time frame, with the majority of recycling potentially routing through the large hepatic TAG pool (16). The serum NEFA used for VLDL TAG achieved a pseudosteady state after a single day of labeling, and this observation supports the idea of the presence of a fast hepatic turnover

\section{Figure 3}

Comparison of sources of TAG fatty acids in liver and lipoprotein fractions. (A and B) Sources of palmitate in VLDL, tTRL, and liver TAG accounted for after 5 days of labeling in subjects 1-9 (A), and the correlation between the percentage of TAG accounted for in the liver and VLDL (B). For both VLDL and TTRL, fasted-state values represent the average source contribution on day 5 at 0700 in subjects $1-9$. Source contributions to TTRL TAG in the fed state were determined using the average values obtained on day 4 at 1500 in subjects $1-9$. For comparison of liver TAG and VLDL TAG, fatty acid sources were normalized to $100 \%$. Subject 3 has been omitted from this analysis due to undetectable concentrations of TAG in liver. pool through which serum NEFAs are routed (see center droplet in liver schematic, Figure 1). The significantly higher slope of the incorporation of NEFA into lipoproteins than those for the incorporation of dietary and DNL fatty acids supports the idea of differential handling of this source in the hepatocyte. The accrual into VLDL TAG of de novo fatty acids could be due to an increase in hepatic pools (i.e., those made locally and packaged into VLDL) or due to the accrual of fatty acids synthesized in the adipose that came to the liver through the serum NEFA pool, although the latter mechanism is less likely, given the 4-fold higher presence of DNL fatty acids in VLDL than in the serum NEFA pool. The lower daily rates of accrual for dietary and DNL fatty acids over the 5 days suggest that these fatty acids enter a hepatic holding pool

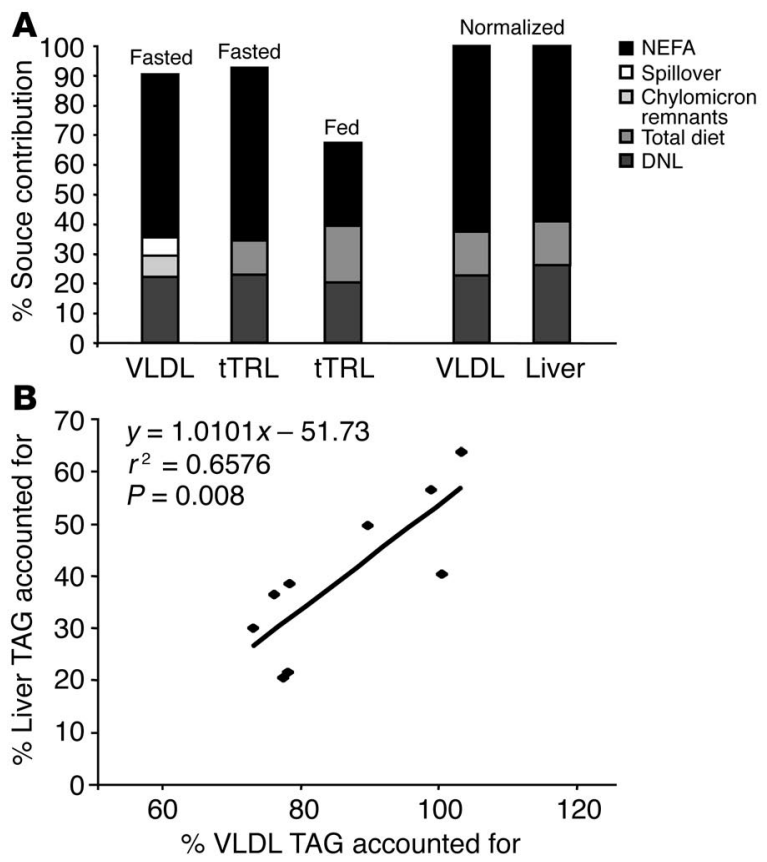


that prevents them from being in immediate communication with the pool of fatty acids used for VLDL TAG synthesis. Moreover, as the final proportions of fatty acid sources in VLDL and liver were similar, it is likely that the liver TAG pool handles these sources of fatty acids in a manner similar to that of those newly made.

A number of limitations of this study deserve mention. The first is that the duration of isotope labeling was only sufficient to account for at most $64 \%$ of the sources of liver TAG fatty acids in any one subject. The duration of 5 days was chosen from a practical standpoint as being the longest time that was feasible for patients to be absent from work to undergo continuous labeling while staying as inpatients at the General Clinical Research Center (GCRC). Second, as ethically only 1 liver biopsy could be performed, it was impossible to know where on a physiologic turnover curve the liver TAG was being sampled. Nonetheless, the estimate of hepatic TAG turnover time $\left(5 \times t_{1 / 2}\right)$ ranged from 20 to 60 days, which was surprisingly long, given estimates of 1-2 days for liver TAG turnover in healthy subjects $(16,39,41,42)$. In contrast, for VLDL TAG, 5 days of labeling was sufficient time to allow for $100 \%$ of the fatty acids to be accounted for in 5 of the 9 subjects. In the 4 subjects still secreting unlabeled lipoprotein fatty acids at the day- 5 time point, the origin of this cold pool could not be definitively identified. Visceral fatty acids can dilute the serum NEFA label that would not be accounted for when sampled peripherally. This would cause an underestimation of the contribution of serum NEFA to VLDL TAG and result in the inability to account for all VLDL fatty acids (a visceral dilution effect). However, we found a strong positive correlation between the percentage of fatty acids accounted for in VLDL TAG and those accounted for in liver TAG suggesting that the origin of unlabeled VLDL fatty acids was not due to visceral dilution but due to use of a cold (unlabeled) hepatic TAG pool. Third, our study lacked a control group, because elective liver biopsy in a control population was not an option. Thus, whether the pattern of fatty acid sources would have been similar in the livers of subjects without NAFLD is unknown. However, the change in fatty acid sources found in VLDL TAG in both the fasting and fed states was compared with that from previous studies $(9,16,33,34,39,41,42)$ and recent data from our lab $(23,36,37)$. An inability to account for all of the fatty acids in VLDL TAG has been observed previously in hypertriglyceridemics (9). Furthermore, given our data (23) and other data in the literature (34), the lack of increase in lipogenesis postprandially in NAFLD is striking. Finally, the goals of our study were to determine the sources of fatty acids utilized for liver and VLDL TAG synthesis in NAFLD, and measurement of TAG or particle production rates was not undertaken. However, data from Charlton et al. have suggested that lipoprotein particle secretion rate (as assessed by apoB turnover) is significantly reduced in nonalcoholic steatohepatitis (43). Furthermore, using $\left[{ }^{13} \mathrm{C}_{1}\right]$ palmitate infusion, Diraison et al. have demonstrated normal TAG secretion rates in NAFLD (18). In the context of those studies, fatty liver could be caused either by low particle secretion rates or by an amount of secreted TAG per particle that is insufficient to keep up with liver TAG synthesis. If either of these events contributed to the fatty liver observed in our patients, then elevated DNL would exacerbate the situation. Our data have shown that elevated lipogenesis provides a significant source of the fatty acids accumulating in the livers of these patients.

In summary, our data provide a quantitative description of the fatty acid sources that contributed to both the liver and lipoprotein TAG of NAFLD patients after 5 days of labeling. Efforts to treat NAFLD by improving insulin sensitivity to reduce peripheral fatty acid flux should be expanded to modulate liver lipogenesis through dietary and pharmacological therapy. With obesity as a key risk factor for NAFLD, and with the recent recognition of the ability of simple sugars to stimulate the lipogenic pathway (33), dietary therapy that advocates the consumption of complex carbohydrates deserves emphasis. One of the underlying causes of fat accumulation in NAFLD is the inability of the liver to regulate the changes in lipogenesis that should occur during the transition from the fasted to the fed state.

\section{Methods}

Subjects. Men and women scheduled for a liver biopsy for diagnosis of NAFLD were recruited by participating physicians at the clinics of Minnesota Gastroenterology (Minneapolis, Minnesota, USA). Patients were not referred for the study if alcohol consumption exceeded $20 \mathrm{~g} /$ wk or if they tested positive for viral hepatitis, Wilson disease, or any other possible cause of liver dysfunction. The subjects' liver function was further assessed through measurement of $\gamma$-globulins (A, G, E, and $M$ ), $\alpha$-1-antitrypsin, $\alpha$-fetoprotein, ferritin, ceruloplasmin, iron, transferrin, and hepatitis A, B, and C. For the present study, potential NAFLD subjects were screened on 2 occasions to obtain a detailed biochemical and nutritional assessment. Patients were included in the study if their physician suspected they had fatty liver, if they had elevated liver enzymes upon initial screening, and if they were not in a period of active weight loss (defined as weight loss exceeding $0.5 \mathrm{~kg} / \mathrm{wk}$ ). There were no restrictions on gender, smoking status, age, or glucose tolerance. Women participants were studied during the luteal phase of the menstrual cycle. All subjects gave written informed consent to participate in this research study, which was approved by the Institutional Review Board of the University of Minnesota (protocol 0106M01801). All medical procedures were performed at the GCRC at Fairview University Medical Center (Minneapolis, Minnesota, USA).

Procedures. Computed tomography scans without contrast were performed on each participant to quantify visceral and subcutaneous fat mass (44). To ensure metabolic steady state, 3 days prior to the 5-day inpatient stay, subjects were instructed to consume a defined eucaloric diet. This diet was based on previously acquired food records and interviews conducted during screening. Subjects recruited were required to be maintaining their weight and to be eating diets of typical composition (55\% carbohydrate, $30 \%$ fat, and $15 \%$ protein). The prescribed diets consumed during the 3-day run-in phase were matched to the subjects' background diet; thus, the subjects were deemed to be in stable metabolic states. Energy requirements were determined by the Harris-Benedict equation with an appropriate activity factor. Subjects' weights were recorded daily to ensure weight maintenance throughout the inpatient protocol. To trace the sources of lipoprotein and hepatic TAG fatty acids in both fasted and fed states, we began isotope infusions 4 days prior to and continued them through each patient's scheduled liver biopsy. $\left[{ }^{13} \mathrm{C}_{1}\right]$ Sodium acetate, $\left[1,2,3,4{ }^{13} \mathrm{C}_{4}\right]$ potassium palmitate, and $\left[{ }^{2} \mathrm{H}_{31}\right]$ glyceryl-tripalmitin were purchased from Cambridge Isotope Laboratory and Isotec. Isotopic purity was greater than $98 \%$ for all tracers used. Deuterated tripalmiate was used to determine the contribution of dietary fatty acids to hepatic and lipoprotein TAG; unpublished data from our lab support the use of this isotope in tracking unlabeled dietary fatty acids into the chylomicron fraction (M.T. Timlin and E.J. Parks, unpublished observations). To measure the contribution of serum NEFA to lipoprotein and liver TAG, we began a constant infusion of $\left[{ }^{13} \mathrm{C}_{4}\right]$ potassium palmitate at a rate of $2.5 \mu \mathrm{g} / \mathrm{kg} / \mathrm{min}$ on day 1 at 1700 . Fatty acid synthesis was measured using a continuous infusion of sodium $\left[{ }^{13} \mathrm{C}_{1}\right]$ acetate $(10 \mathrm{~g} / \mathrm{d})$, also beginning at 1700 on day 1 . The contribution of dietary TAG to liver and lipoprotein 
pools was measured using $\left[{ }^{2} \mathrm{H}_{31}\right]$ glyceryl-tripalmitin (1-2 g/d), which was administered in whole food with each meal eaten at the GCRC. In both the fasted state (0645) and fed state (1500), blood was drawn intermittently over the 5-day study period and lipoproteins were isolated each day. On day 4 of the protocol, subjects underwent a meal test to determine lipid flux during the postprandial period. At 0700, a breakfast was consumed consisting of whole foods (30\% fat, $55 \%$ carbohydrate, and $15 \%$ protein). Of the grams of carbohydrate provided in the meal, $5.3 \%, 5.6 \%, 8.6 \%, 6.2 \%$, and $53.4 \%$ were from glucose, fructose, lactose, sucrose, and starch, respectively, with the remainder of carbohydrate weight provided by dietary fiber. A fasting blood sample was obtained prior to the meal, and blood samples were collected immediately after meal consumption for every 15 minutes until 0800 and hourly thereafter. Indirect calorimetry was performed twice for 30 minutes for the assessment of substrate oxidation in the fasted and fed states using a Deltratrac II metabolic cart in the hooded mode (Sensor Medix). All blood was drawn into iced Vacutainers containing $1 \mathrm{mg} / \mathrm{ml}$ EDTA. Serum was separated immediately by centrifugation $\left(1,500 \mathrm{~g}\right.$ for 20 minutes) at $4^{\circ} \mathrm{C}$, kept on ice, and separated into aliquots for various analyses. The liver biopsy occurred at the GCRC at 0800 on day 5 and was performed by the referring physician. Approximately 1 hour prior to the biopsy, patients were given an intramuscular injection of $100 \mathrm{mg}$ meperidine hydrochloride (Abbott Laboratories) plus $50 \mathrm{mg}$ hydroxyzine hydrochloride (American Regent).After the biopsy, $15 \mathrm{mg}$ of the liver tissue was set aside for analysis of TAG sources. Histological evaluation of all biopsies was performed by the same pathologist, and samples were graded for the presence of fat infiltration, necroinflammation, and fibrosis according to the grading system of Dixon (45).

Lipoprotein isolation, lipid extraction, and laboratory analysis. To determine whether the sources of TAG fatty acids stored in the liver were utilized for TAG exported out of the liver, we isolated lipoproteins from serum as described previously (9). Briefly, tTRLs (density $<1.006 \mathrm{~g} / \mathrm{ml}$ ) were isolated from serum by ultracentrifugation for 20 hours in a 50.3 Beckman rotor $\left(1.3 \times 10^{8} \mathrm{~g}\right.$ at $\left.10^{\circ} \mathrm{C}\right)$. The tTRL fraction (upper $2 \mathrm{ml}$ ) was collected by tube slicing. For fed-state samples, tTRLs alone were analyzed. Samples collected in the fasting state were dialyzed for 48 hours to a density of $1.21 \mathrm{~g} / \mathrm{ml}$ and were subjected to further lipoprotein fractionation. Briefly, lipoproteins with an $\mathrm{S}_{\mathrm{f}}$ greater than 400 were separated by ultracentrifugation, and after that, the lipoproteins with an $\mathrm{S}_{\mathrm{f}}$ of $60-400$ were separated. The lipoproteins with an $\mathrm{S}_{\mathrm{f}}$ of $60-400$ represent a fraction with large VLDL particles (diameter, $70-400 \mathrm{~nm}$ ) that are TAG rich and have a very short half-life (46). After the addition of a lipid internal standard to the lipoprotein samples with an $\mathrm{S}_{\mathrm{f}}$ of $60-400$, total lipids were extracted from plasma lipoproteins and also from liver tissue by the Folch method (47). NEFAs were extracted immediately from serum with the addition of 30:70 ( $\mathrm{vol} / \mathrm{vol}$ ) heptane/isopropanol. Individual lipids from tissue, serum, tTRL and lipoproteins with an $\mathrm{S}_{\mathrm{f}}$ of 60-400 were separated, the TAG and NEFA transesterified (9), and the fatty acid methyl esters were analyzed by gas chromatography and gas chromatography/ mass spectrometry (GC/MS) using palmitic acid (16:0) as the marker of fatty acid metabolites. Concentrations of glucose in serum samples were measured with a Vitros Analyzer 950 (Ortho-Clinical Diagnostics) and concentrations of insulin were determined by chemiluminescence immunoassay (Diagnostic Products Corporation). Serum TAG and NEFA concentrations were determined by enzymatic assay (Wako Chemicals USA Inc.) with a microtiter spectrophotometer (Model EL 340 Microplate; Bio-Tek Instruments Inc.). For determination of the enrichment of label in the total dietary fat, all foods consumed on a single day were placed in a blended. The enrichment of the TAG palmitate of this composite food was quantified by GC/MS and was shown to be $9.9 \% \pm 4.9 \%$. Lipoproteins were fractionated from serum collected at 0645 on days
$2,3,4$, and 5 for the fasting samples and at 1500 for postprandial samples. GC/MS was performed on an HP 6890 with a Mass Selective Detector HP 5973 fitted with an ETP electron multiplier (SGE Inc.) using a HP-1 $25-\mathrm{m}$ column, with a $250-\mu \mathrm{m}$ inner diameter and $0.33-\mu \mathrm{m}$ film thickness and helium as the carrier gas. Electron impact was used to selectively monitor ions with mass/charge $(m / z)$ ratios of 270,271 , $272,274,300$, and 301. Comparable ion peak areas between standards and biological samples were achieved by either adjustment of the volume injected or by dilution or concentration of the sample. The TAG concentrations in liver samples were determined using an HP6890 gas chromatograph (Hewlett Packard). The gas chromatograph was fitted with an automatic split-injection system and a flame-ionization detector. A fused silica capillary column was used $(50 \mathrm{~m}$ in length, internal diameter of $0.18 \mu \mathrm{m}, 007-23$; Quadrex Corp.), and individual fatty acids were identified by retention time and were compared with an internal standard for quantification.

Isotope methodology, calculations, and statistics. By tracking the variously labeled methyl-palmitate isotopomers $\left(\mathrm{M}_{0}, \mathrm{M}_{1}, \mathrm{M}_{2}, \mathrm{M}_{4}, \mathrm{M}_{30}\right.$, and $\left.\mathrm{M}_{31}\right)$ in liver TAG and VLDL TAG using GC/MS, information regarding the sources of fatty acids used for TAG synthesis was obtained. These data are presented as the proportion of fatty acids derived from the three sources (i.e., data are presented as percentage of fatty acids in VLDL TAG from NEFAs, lipogenesis, and diet). In liver and lipoproteins, DNL was calculated using mass isotopomer distribution analysis (MIDA) $(48,49)$. MIDA is a technique based on combinatorial probabilities, with the central principle being that the isotopic enrichment of the molecule of interest is directly dependent on the enrichment of the precursor pool used for its synthesis. Thus, the labeling pattern in the precursor pool is determined by the isotopomer pattern of the polymer. Dietary and NEFA enrichments of both liver and lipoproteins were calculated using a 5-point standard curve. Comparable ion peak areas between the standard curve and biological samples were achieved by either dilution or concentration of the sample. The RaNEFA into the serum NEFA pool was calculated using the steady-state NEFA enrichment obtained on the morning of day 2 (approximately 12 hours after the start of infusion). At later time points it was anticipated that NEFA recycling would reduce the accuracy of this measurement. The infusate composition and enrichment were also analyzed by GC and GC/MS and the calculations were adjusted for the amount of unlabeled fatty acids present that were derived from less than $100 \%$ isotopic purity and from fatty acids present on the albumin used in the infusion. The sources of serum NEFA as shown in Figure $2 \mathrm{~A}$ are adipose, DNL, and diet, although it should be pointed out that dietary fatty acids in the NEFA pool are designated as originating in chylomicron spillover. Our methodology cannot discern whether these fatty acids actually entered adipose and were rereleased into the serum NEFA pool. Furthermore, newly made fatty acids in the serum NEFA pool could have been synthesized de novo in either adipose tissue (and released after TAG lipolysis) or liver (and derived from VLDL spillover). The RaNEFA discussed here takes into account the input of unlabeled dietary fatty acids that spilled over into the serum NEFA pool. Spillover fatty acids were subtracted from the NEFA concentration, then adipose contribution was then calculated using this equation for corrected $\mathrm{M}_{4}$.

\section{Equation 1}

$\%\left[{ }^{13} \mathrm{C}_{4}\right]$ hexadecanoate in NEFA

$1-\left(\frac{\%\left[\mathrm{~d}_{31}\right] \text { hexadecanoate in NEFA }}{\%\left[\mathrm{~d}_{31}\right] \text { hexadecanoate in dietary TAG }}+\%\left[{ }^{13} \mathrm{C}_{4}\right]\right.$ hexadecanoate in NEFA $)$

where $\mathrm{d}$ indicates deuterium. 
The percentage of VLDL TAG palmitate derived only from adipose tissue release is calculated by Equation 2 .

\section{Equation 2}

$\frac{\%\left[{ }^{13} \mathrm{C}_{4}\right] \text { hexadecanoate in VLDL TAG }}{\text { corrected } \mathrm{M}_{4}}$

The percentage of VLDL TAG from diet-derived NEFA (the spillover pathway) is calculated by Equation 3 .

\section{Equation 3}

$\left(\frac{\%\left[\mathrm{~d}_{31}\right] \text { hexadecanoate in NEFA }}{\%\left[{ }^{13} \mathrm{C}_{4} \text { hexadecanoate in NEFA }\right.}\right) \times \%\left[{ }^{13} \mathrm{C}_{4}\right]$ hexadecanoate in VLDL TAG $\%\left[d_{31}\right]$ hexadecanoate in dietary TAG

Among all subjects, it was observed that the ratio of $\left[\mathrm{d}_{31}\right]$ palmitate to $\left[{ }^{13} \mathrm{C}_{4}\right]$ palmitate was always greater in VLDL TAG than in the serum NEFA pool. This additional source of dietary fatty acids must be from chylomicron-remnant TAG that entered the liver either directly via remnant receptor uptake or indirectly via transfer to HDL, or via lipolysis at the hepatocyte. Thus, given Equation 3, the percentage of VLDL TAG derived from hepatic uptake of chylomicron-remnant TAG is calculated by subtracting the portion of dietary fatty acids derived via the spillover pathway from the total contribution of dietary fatty acids to VLDL TAG.

VLDL TAG derived from chylomicron-remnant TAG is calculated by Equation 4.

\section{Equation 4}

$\%\left[d_{31}\right]$ hexadecanoate in VLDL TAG

$\%\left[\mathrm{~d}_{31}\right]$ hexadecanoate in dietary TAG - Equation 3

Liver turnover data were calculated using an assumed liver weight of $2,000 \mathrm{~g}$ and an estimation of $5 \times t_{1 / 2}$ for near-complete turnover of the hepatic TAG pool (50). Differences in source contribution over time were analyzed using repeated-measures analysis of variance. Statistical differences between fasting and feeding and between sources of VLDL TAG palmitate and liver TAG palmitate were determined by paired Student's $t$ tests using Statview (version 5.0.1.; SAS Institute Inc.). Correlations were analyzed using simple regression. A $P$ value of less than 0.05 was considered significant.

\section{Acknowledgments}

This research was supported by grants from the NIH (PAR-98086) and the National Center for Research Resources/NIH General Clinical Research Center Program (M01-RR00400). We are grateful to the participants for contributing their time to the study; to Audrey Schieber for providing laboratory assistance; and to the staff at Fairview-University Medical Center, General Clinical Research Center, and Investigational Pharmacy for their skilled clinical assistance. In particular, the contribution of Mary Coe was much appreciated. We especially thank Richard Neese for early support in establishing mass spectrometry techniques and Richard J. Havel and J. Bruce German for insightful discussions on the data.

Received for publication October 14, 2004, and accepted in revised form February 1, 2005.

Address correspondence to: Elizabeth J. Parks, Department of Food Science and Nutrition, University of Minnesota, 1334 Eckles Avenue, St. Paul, Minnesota 55108, USA. Phone: (612) 625-1785; Fax (612) 625-5272; E-mail: eparks@umn.edu.

Authors of this work are members of the Lipid Research Group at the University of Minnesota, Departments of Medicine and Food Science and Nutrition, Twin Cities, St. Paul, Minnesota, USA.

Portions of this work were presented in a symposium at the American Diabetes Association National Meeting in Orlando, Florida, USA, on June 6, 2004, and at the American Association for the Study of Liver Disease Liver Meetings in Boston, Massachusetts, USA, on November 31, 2004.
1. Ludwig, J., Viggiano, T.R., McGill, D.B., and Oh, B.J. 1980. Nonalcoholic steatohepatitis: Mayo Clinic experiences with a hitherto unnamed disease. Mayo Clinic. Proc. 55:434-438.

2. Clark, J.M., Brancati, F.L., and Diehl, A.M. 2002. Nonalcoholic fatty liver disease. Gastroenterology. 122:1649-1657.

3. Lee, R.G. 1989. Nonalcoholic steatohepatitis: a study of 49 patients. Hum. Path. 20:594-598.

4. Bacon, B.R., Farahvash, M.J., Janney, C.G., and Neuschwander-Tetri, B.A. 1994. Nonalcoholic steatohepatitis: an expanded clinical entity. Gastroenterology. 107:1103-1109.

5. James, O., and Day, C. 1999. Non-alcoholic steatohepatitis: another disease of affluence. Lancet. 353:1634-1636.

6. Sanyal, A.J. 2002. AGA technical review on nonalcoholic fatty liver disease. Gastroenterology. 123:1705-1725.

7. Marchesini, G., et al. 1999. Association of nonalcoholic fatty liver disease with insulin resistance. $\mathrm{Am}$. J. Med. 107:450-455.

8. Marchesini, G., et al. 2001. Nonalcoholic fatty liver disease: a feature of the metabolic syndrome. Diabetes. 50:1844-1850

9. Parks, E.J., Krauss, R.M., Christiansen, M.P., Neese, R.A., and Hellerstein, M.K. 1999. Effects of a lowfat, high-carbohydrate diet on VLDL-triglyceride assembly, production and clearance. J. Clin. Invest. 104:1087-1096.

10. Chen, Y.I., Golay, A., Swislocki, A.L.M., and Reaven, G.M. 1987. Resistance to insulin suppression of plasma free fatty acid concentrations and insulin stimulation of glucose uptake in noninsulindependent diabetes mellitus. J. Clin. Endocrinol. Metab. 64:17-21.

11. Kooner, J.S., et al. 1998. Abdominal obesity, impaired nonesterified fatty acid suppression, and insulin-mediated glucose disposal are early metabolic abnormalities in families with premature myocardial infarction. Arterioscler. Thromb. Vasc. Biol. 18:1021-1026.

12. Frayn, K.N., and Coppack, S.W. 1992. Insulin resistance, adipose tissue and coronary heart disease. Clin. Sci. 82:1-8.

13. Sanyal, A.J., et al. 2001. Nonalcoholic steatohepatitis: association of insulin resistance and mitochondrial abnormalities. Gastroenterology. 120:1183-1192.

14. Miles, J.M., et al. 2004. Systemic and forearm triglyceride metabolism: fate of lipoprotein lipasegenerated glycerol and free fatty acids. Diabetes. 53:521-527.

15. Havel, R.J., and Hamilton, R.L. 2004. Hepatic catabolism of remnant lipoproteins: where the action is. Arterioscler. Thromb. Vasc. Biol. 24:213-215.
16. Farquhar, J.W., Gross, R.C., Wagner, R.M., and Reaven, G.M. 1965. Validation of an incompletely coupled two-compartment nonrecycling caternary model for turnover of liver and plasma triglyceride in man. J. Lipid Res. 6:119-134.

17. Reaven, G.M., Hill, D.B., Gross, R.C., and Farquhar, J.W. 1965. Kinetics of triglyceride turnover of very low-density lipoproteins in human plasma. J. Clin. Invest. 44:1826-1833.

18. Diraison, F., Moulin, P.H., and Beylot, M. 2003. Contribution of hepatic de novo lipogenesis and reesterification of plasma nonesterified fatty acid to plasma triglyceride synthesis during non-alcoholic fatty liver disease. Diabetes Metab. 29:478-485.

19. Donnelly, K.L., Margosian, M.R., Sheth, S.S., Lusis, A.J., and Parks, E.J. 2004. Increased lipogenesis and fatty acid reesterification contribute to hepatic triacylglycerol stores in hyperlipidemic Txnip-/mice. J. Nutr. 134:1475-1480.

20. Donnelly, K.L., Smith, C.I., Schwarzenberg, S.J., Boldt, M.D., and Parks, E.J. 2004. Stable isotope methodology to identify sources of fat in liver biopsy tissue from patients with non-alcoholic fatty liver disease. Diabetes. 56:A53.

21. Dixon, A.K. 1983. Abdominal fat assessed by computed tomography: sex difference in distribution. Clin. Radiol. 34:189-191.

22. Matthews, D.R., et al. 1985. Homeostasis model 
assessment: insulin resistance and B-cell function from fasting plasma glucose and insulin concentrations in man. Diabetologia. 28:412-419.

23. Timlin, M.T., and Parks, E.J. 2005. The temporal pattern of de novo lipogenesis in the postprandial state. Am. J. Clin. Nutr. 81:35-42.

24. Horton, J.D., Goldstein, J.L., and Brown, M.S. 2002. SREBPs: activators of the complete program of cholesterol and fatty acid synthesis in the liver. J. Clin. Invest. 109:1125-1131. doi:10.1172/ JCI200215593.

25. Laurell, S., and Lundquist, A. 1971. Lipid composition of human liver biopsy specimens. Acta Med. Scand. 189:65-68.

26. Martinsson, A., Sunzell, H., and Hood, B. 1963. Nitrogen, lipid, glycogen and deoxyribonucleic acid content of human liver. Acta Med. Scand. 173:745-752.

27. Marchesini, G., et al. 2001. Metformin in non-alcoholic steatohepatitis. Lancet. 358:893-894.

28. Caldwell, S.H., et al. 2001. A pilot study of a thiazolidinedione, troglitazone, in nonalcoholic steatohepatitis. Am. J. Gastroenterol. 96:519-525.

29. Vajro, P., et al. 1994. Persistent hyperaminotransferasemia resolving after weight reduction in obese children. J. Pediatr. 125:239-241.

30. Bajaj, M., et al. 2003. Pioglitazone reduces hepatic fat content and augments splanchnic glucose uptake in patients with type 2 diabetes. Diabetes. 52:1364-1370.

31. Promrat, K., et al. 2004. A pilot study of pioglitazone treatment for nonalcoholic steatohepatitis. Hepatology. 39:188-196.

32. Schwarz, J.-M., Linfoot, P., Dare, D., and Aghajanian, K. 2003. Hepatic de novo lipogenesis in normoinsulinemic and hyperinsulinemic subjects consuming high-fat, low-carbohydrate and low-fat, high-carbohydrate isoenergetic diets. Am. J. Clin. Nutr. 77:43-50.

33. Parks, E.J. 2002. Dietary carbohydrate's effects on lipogenesis and the relationship of lipogenesis to blood insulin and glucose concentrations. $\mathrm{Br}$. J. Nutr. 87(Suppl. 2):S247-S253.

34. Hudgins, L.C., et al. 2000. Relationship between carbohydrate-induced hypertriglyceridemia and fatty acid synthesis in lean and obese subjects. J. Lipid Res. 41:595-604.

35. Miles, J.M., et al. 2004. Systemic and forearm triglyceride metabolism: fate of lipoprotein lipasegenerated glycerol and free fatty acids. Diabetes. 53:521-527.

36. Barrows, B.R., and Parks, E.J. 2004. Fatty acid contribution to very-low density lipoprotein triacylglycerol (VLDL-TG) during fasted and postprandial states in healthy males. Diabetes. 56:A1388.

37. Timlin, M.T., and Parks, E.J. 2004. Study of fatty acid sources contributing to VLDL-triglyceride (TG) postprandially using multiple stable isotopes. Diabetes. 56:A338.

38. Baar, R.A., et al. 2005. Investigation of in vivo fatty acid metabolism in AFABP/aP2 -/- mice. Am. J. Physiol. 288:E187-E193.

39. Baker, N. 1969. The use of computers to study rates of lipid metabolism. J. Lipid Res. 10:1-24.

40. Patterson, B.W. 1997. Use of stable isotopically labeled tracers for studies of metabolic kinetics: an overview. Metabolism. 46:322-329.

41. Boberg, J., Carlson, L.A., and Freyschuss, U. 1972. Determination of splanchnic secretion rate of plasma triglycerides and of total and splanchnic turnover of plasma free fatty acids in man. Eur. J. Clin. Invest. 2:123-132.

42. Nilsson, S., and Schersten, T. 1969. Synthesis of phospholipids and triglyceride in human liver slices. Scand. J. Clin. Lab. Invest. 24:237-249.

43. Charlton, M., Sreekumar, R., Rasmussen, D., Lindor, K., and Nair, K.S. 2002. Apolipoprotein synthesis in nonalcoholic steatohepatitis. Hepatology. 35:898-904.

44. Yoshizumi, T., et al. 1999. Abdominal fat: standardized technique for measurement at CT. Radiology. 211:283-286.

45. Dixon, J.B., Bhathal, P.S., and O'Brien, P.E. 2001. Nonalcoholic fatty liver disease: predictors of nonalcoholic steatohepatitis and liver fibrosis in the severely obese. Gastroenterology. 121:91-100.

46. Marzetta, C.A., Foster, D.M., and Brunzell, J.D. 1990. Conversion of plasma VLDL and IDL precursors into various LDL subpopulations using density gradient ultracentrifugation. J. Lipid Res. 31:975-984.

47. Folch, J., Lees, M., and Sloane Stanley, G.H. 1957. A simple method for the isolation and purification of total lipids from animal tissues. J. Biol. Chem. 226:497-509.

48. Hellerstein, M.K., Neese, R.A., and Schwarz, J.M. 1993. Model for measuring absolute rates of hepatic de novo lipogenesis and reesterification of free fatty acids. Am. J. Physiol. 265:E814-E820.

49. Hellerstein, M.K. 1995. Methods for measurement of fatty acid and cholesterol metabolism. Curr. Opin. Lipidol. 6:172-181.

50. Wolfe, R.R. 1992. Radioactive and stable isotope tracers in biomedicine. John Wiley \& Sons Inc. New York, New York, USA. 471 pp. 